\title{
What we expect of integrative medicine case reports?
}

\author{
Subhash Chandra Lakhotia \\ Cytogenetics Laboratory, Department of Zoology, Banaras Hindu University, Varanasi 221005, India
}

KEY WORDS

Case report

Integrative medicine

*Corresponding Author:

Subhash Chandra Lakhotia, PhD

Cytogenetics Laboratory

Department of Zoology

Banaras Hindu University

Varanasi 221005, India.

E-mail: lakhotia@bhu.ac.in

In recent times, launching of new research Journals has become common and also worrisome. Increasing demands and specializations require new research Journals. Thus while new Journals are welcome, the worry stems from the fact that a majority of the newly launched Journals have unscrupulous commercial interests rather than their being interested in sharing of good new knowledge between peers. Thus we need to ask why is this new Journal required and what we expect from it? When Prof. Akshay Anand, the Editor-in-Chief of this Journal asked me to write an editorial for the first issue, I was a little surprised. I am not trained in Ayurveda, Unani or any of the other traditional or modern healthcare system. The only connection that I have with these alternative medical systems is that one of my research areas relates to basic research in Ayurvedic Biology using the Drosophila model. My other qualification for writing this editorial can be my longterm conviction that Indian academia need to publish good research Journals and that our researchers should take pride in publishing in such Journals also.

Ayurveda or the other traditional health-care systems in India have been practiced for several thousand years. Ayurveda practitioners are justifiably grateful to the ancient scholars who compiled remarkably detailed principles, philosophies and treatment regimens thousands of years ago, which have been practiced since then. Therefore, Ayurveda practitioners generally argue that these practices, unlike the recent modern medical practices, do not need any validation, experience having established their usages. Such an argument is flawed and may indeed be a stumbling block for a much wider acceptance of the AYUSH system. The diverse formulations and practices used in Ayurveda on basis of experience indeed need unbiased experimental and clinical validation. Despite antiquity and wide usages, it is to be appreciated that climate, flora, fauna and our own life-styles etc have undergone substantial changes over the past millennia. Since Ayurveda considers the physical human body, its mental state and its relation with abiotic and biotic environment (internal and external) to be intimately linked and therefore, each of them impacts not only health but also the required therapy. Consequently, the various therapeutic approaches prescribed in classical Samhitas may need re-examination in the context of current life styles and the much better understanding of biological systems including human physiology. Ayurveda, Yoga and other traditional medicine practitioners should appreciate that a re-examination and revalidation of old principles and practices is not to question the wisdom of the ancient scholars. Such studies would in fact only be following their dictum that textual narrations must be re-examined and re-validated before being accepted.

It has often been felt that the traditional and modern health-care systems should be integrated, taking the best from each so that patients get a more holistic and effective care. In this context, this Journal's policy to also publish combinatorial effects of modern medicine and traditional approaches as well as admixture of AYUSH, is significant.

Many of the published case-studies reports in modern or traditional health-science lack the required levels of unbiased approach, experimental rigor and statistical power. In the traditional medicine systems, it is all the more important that objective of the studies is not to confirm the common beliefs about a given therapy. It should rather be driven by an attitude of unbiased enquiry. While we need to appreciate the ancient wisdom, the recent trend of claiming a 'know all' feature of ancient scholars and their creative works can be very counterproductive. It is often claimed that ancient scriptures in India are full of information/concepts that much of the current civilization believes to have been understood only in recent times. Even if this were true, it still does not give any practical advantage unless we learn the mechanistic bases of such claimed concepts/propositions. This necessitates 
unbiased re-examination so that myths can be separated from facts. This applies to all the traditional health-care systems as well. It is expected that the availability of this Journal devoted primarily to traditional medicine case reports would encourage more systematic and in-depth studies that provide reliable experimental evidence and thus more effective patient-care regimens.

Success of a Journal primarily depends upon the authors who submit their manuscripts and the readers who find the published material worth reading. The other important human component is the Editor and his/her office, which includes the peer-reviewers too. The Editor and peer-reviewers are to ensure certain minimal level of quality in the published material. This is achieved by filtering out the below standard submitted manuscripts and improving upon the others so that readers look forward to reading what is published. And, if they enjoy, they would like to cite the published work in their own research output. The peer-reviewers are an important component in the contemporary research publication chain. They are expected to take a constructive view, with a stringent yard-stick for quality and integrity of the contents while assessing the manuscript referred to them by the Editor. The peerreviewers should provide their comments on the referred manuscript keeping in mind that they are also authors as well as readers. The Editor should not function only like a post-office that transmits comments of reviewers to author and back, an experience that many of us encounter in recent times. Editor, being an informed researcher in the given area, has to also function as an independent reviewer and arbiter, and he/she should take decisions contrary to reviewers' recommendations, if required. This, however, demands more time and energy on the part of the Editor. Readers, besides reading the published articles, also should be able to react and point out any limitations of the given study.

Authors who submit their work to this Journal are expected to follow the desired high standards of analysis using well-designed experimental plan/s. The Editor/s and Reviewer/s too would need to follow the best publication ethics so that this new Journal from India provides the much needed space for rejuvenation of traditional health-care systems. My best wishes to the editorial team, authors and readers for success of this new venture.

doi: 10.38205/imcr.010101 